\title{
EXTENDED PEDICLE RECTUS ABDOMINIS MYOCUTANEOUS FLAP FOR GROIN RECONSTRUCTION: CASE REPORT
}

\author{
S.O. KHAINGA
}

\begin{abstract}
SUMMARY
Reconstruction of left groin defect following a failed left femoral aneurysm bypass procedure. The defect was occasioned by excision of a saccular aneurysm of left femoral artery, and repair with PTFE graft for an ilio femoral bypass. On the second postoperative day, the wound became septic and the graft extruded with a resultant wound defect of about $20 \times 10 \mathrm{~cm}$. The graft was later removed and wound coverage achieved by an extended contralateral pedicled rectus abdominis myocutaneous flap.
\end{abstract}

\section{INTRODUCTION}

A rectus abdominis myocutaneous flap can provide a large amount of tissue for defect coverage. Rarely a flabby and redundant abdominal tissue is used as a huge extended flap, but when applied it can be used in the coverage of defects of anterior thorax, posterior trunk, abdomen, groin and perineum as well as reconstruction of the breast, perineum and vagina (1). As a free flap, it can be used for distant coverage of defects of the head and neck, posterior trunk, upper extremity, lower extremity and as a functional muscle in head and neck. This flap is extremely reliable and any complications are invariably technical in nature due to damage of dominant vessels or shearing stresses with injury to indirect perforator system (2).

\section{CASE REPORT}

A49-year-old man was admitted at Kenyatta National Teaching and Referral Hospital on $10^{\text {th }}$ March 2005 as a referral from Moi Teaching and Referral Hospital with a history of a progressive swelling in the right femoral region. There was no associated history of trauma.

On examination, the patient was sick looking and wasted. There was no pallor, no jaundice, no lymphadenopathy and no pedal oedema. The blood pressure was $100 / 60 \mathrm{mmHg}$. Examination of the respiratory, central nervous and gastrointestinal systems did not reveal any abnormalities. However, local examination in the left inguinal region revealed a femoral swelling, nontender, and pulsatile approximately $15 \times 10 \mathrm{~cm}$. The swelling had started to ulcerate.

An ultrasound done at Moi Teaching and Referral Hospital for the left groin region was suggestive of a dissecting aneurysm of left femoral artery.

The patient was then referred to Kenyatta National Teaching and Referral Hospital for management by a cardiovascular surgeon.

At Kenyatta National Referral and Teaching Hospital, a CT-angiogram with a 3D reconstruction was performed. It revealed a large aneurysm $15.8 \times 13.4 \times 12.3 \mathrm{~cm}$ arising from left ilio femoral artery beginning from within the abdomen, pushing the bladder to the right and extending to the upper thigh. Its posterior and superior portions were filled with a thrombus. The anterior thigh muscles were displaced by the aneurysm and gas was seen within the aneurysm and adjacent muscle planes. The distal femoral artery was patent though slightly smaller in calibre than normal.

A diagnosis of a saccular aneurysm of left femoral artery was made and the patient was taken to theatre for bypass grafting on $14^{\text {th }}$ March 2005, the fourth day after admission at KNH. 
Under general anaesthesia, the aneurysm was accessed via a midline abdominal incision and a $3 \mathrm{~cm}$ abdominal aortic aneurysm was encountered. Proximal control of the left common iliac artery was obtained. Femoral artery was accessed through a medial longitudinal incision in the lower one third of the left thigh and a normal femoral artery found was mobilised by sharp and blunt dissection to gain distal control.

The femoral aneurysm was accessed through a longitudinal incision along anterior aspect of upper one third of left thigh. A large saccular aneurysm of left common femoral artery was found of about $8 \mathrm{~cm}$ diameter with an infected thrombus in its lumen. The thrombus was evacuated, proximal and distal lumen of femoral artery located and embolectomy with a foggarty catheter done to obtain good forward and backward flow. The subcutaneous tissues on the medial aspect of left thigh were found to be infected. In view of this, a decision to perform a right saphenous vein graft was agreed upon and the vein mobilised but its lumen was found to be too small for an ilio femoral bypass graft. As an alternative, a fifteen by sixteen centimetre PTFE graft was used to perform a left ilio femoral bypass graft after cleaning the infected thrombus with hydrogen peroxide, saline and betadine.
The abdominal incision was closed by mass closure and that of the distal end of thigh was closed in layers. The proximal half of wound was packed with betadine gauze to allow for secondary suturing of the wound.

Histology of the excised portion of aneurysmal left femoral artery showed a degenerative blood vessel wall with laminated clot and active chronic inflammation.

On first postoperative day, a pus swab was done and its culture grew proteus spp. and the patient was started on Rocephin. On second postoperative day, wound became very septic and the graft extruded with a slough of surrounding tissues. At this stage, plastic surgeons were consulted and they recommended debridement and removal of the now non-functional bypass graft, pending rotational flap closure once infection settled.

Two months later, $13^{\text {th }}$ June 2005 , the patient was taken to theatre to remove the non-functional graft and an attempt to manipulate the left hip joint which had now developed a flexion contracture failed due to increased bleeding from wound.

On $15^{\text {th }}$ July 2005, four months after the bypass surgery, the plastic surgeons performed an extended contralateral rectus abdominis myocutaneous flap to close the left inguinal wound.

\section{PROCEDURE}

Figure 1

Left groin ulcer measuring $20 \times 10 \mathrm{~cm}$

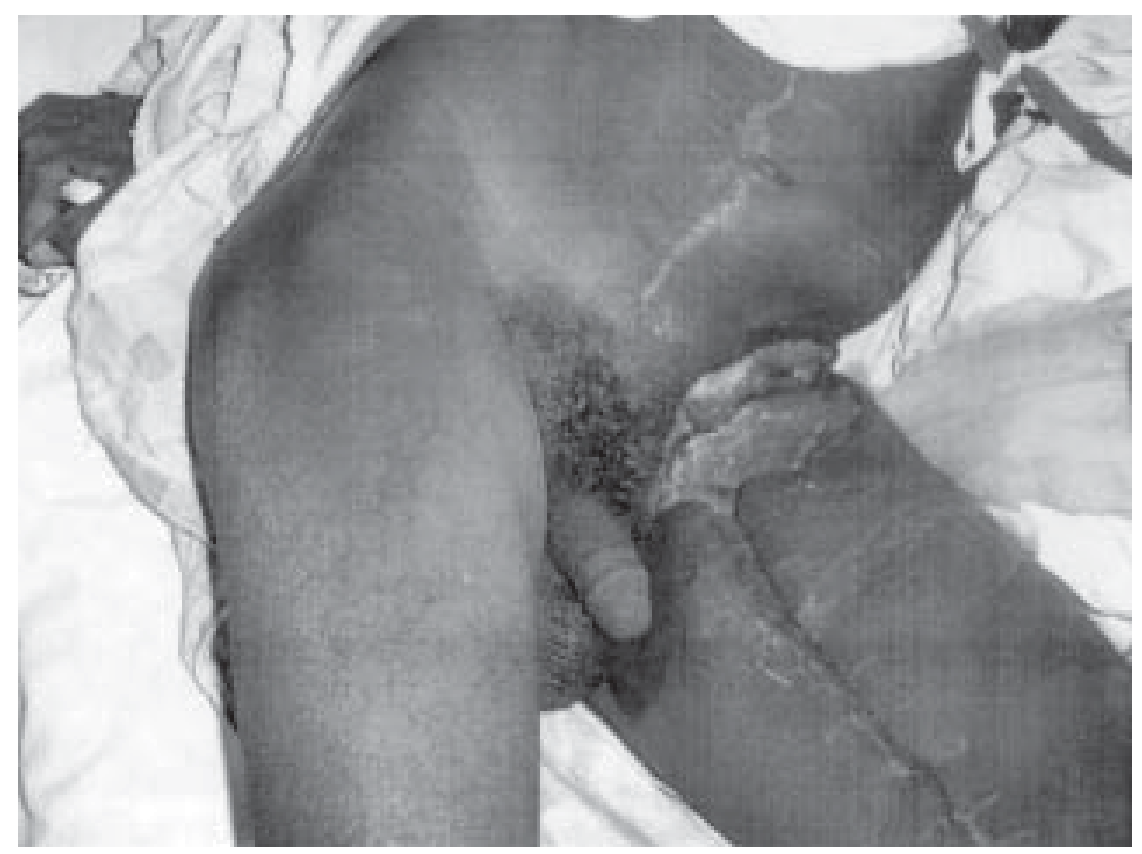




\section{Figure 2}

Transverse island rectus myocutaneous flap designed

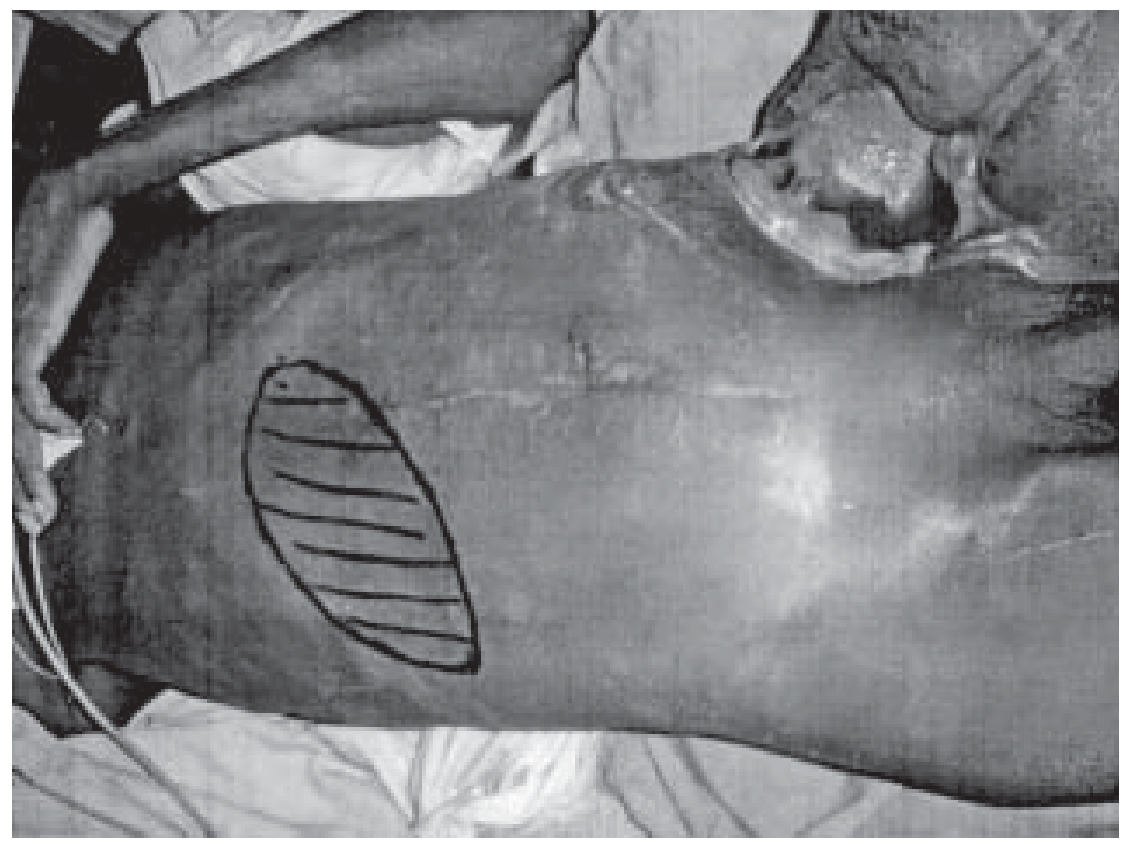

Figure 3

Island rectus myocutaneous flap inset in the left groin defect

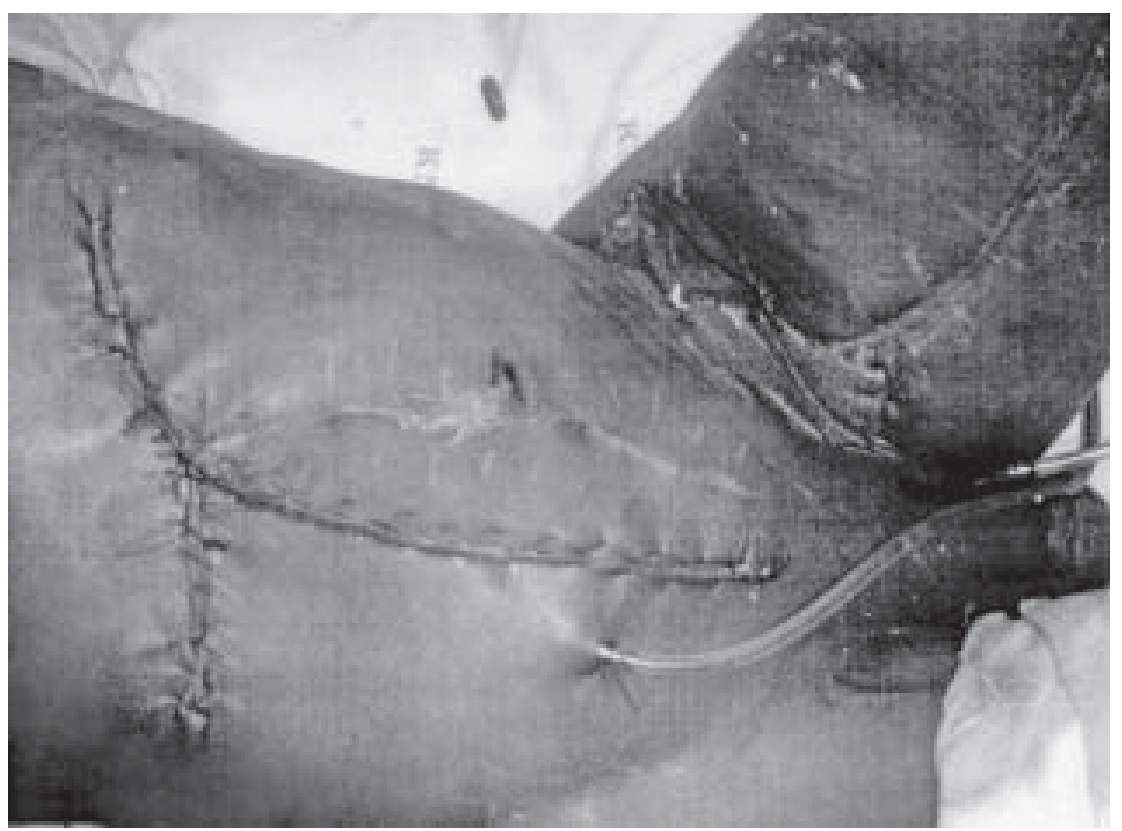

The groin defect that the patient had is as shown in Figure 1. Under general anaesthesia, markings for a transverse skin island for an inferiorly based contralateral rectus abdominis flap were designed (Figure 2) and flap elevated and passed through the subcutaneous tunnel of the lower abdomen to reach the left groin and inset in the already debrided defect (Figure 3). The flap executed at a rotation angle of about $120^{\circ}$. 


\section{RESULTS}

The flap inset was successful and the patient's general status improved. However, two weeks later, a sinus discharging pus from the superior aspect of the flap developed. The patient was taken to theatre for draining of abscess on $21^{\text {st }}$ August 2005. About $400 \mathrm{ml}$ of pus was drained and the sinus was enlarged to allow free drainage of abscess. An abdominal scan done seven days later on $28^{\text {th }}$ August 2005, showed no more accumulation of pus.

The patient was finally discharged back to Moi Teaching and Referral Hospital on $20^{\text {th }}$ October 2005, with a largely reconstructed left groin wound, except for a small discharging sinus and a flexion contracture of left hip.

\section{DISCUSSION}

The rectus abdominis muscle is a vertically oriented abdominal muscle extending between the costal margin and the pubic region. It's a long, flat muscle with three tendinous intersections located at the level of umbilicus, the xiphoid process and mid way between the xiphoid process and umbilicus. The muscle is about $25 \times 6 \mathrm{~cm}$ and its origin is the crest of pubis and the symphysis pubis and inserts via three fascicles into costal cartilages of 5th, 6th and 7th ribs. It is a type III pattern of circulation flap and it can provide a muscle as well as a musculocutaneous flap for wound coverage $(3,4)$.

This flap has two dominant pedicles based on superior and inferior epigastric arteries and veins and minor pedicle based on subcostal and six or seven intercostals arteries and venae comitantes.

The standard skin island is located directly over the surface of the muscle in a vertical orientation. A skin island may also be designed transversely across the abdomen based on musculocutaneous perforating vessels from the rectus muscle $(2,4)$. This was the type of skin island designed for this patient.

This flap can be modified so as to enhance its application;

(i) Preliminary expansion of the lateral abdominal wall skin will increase flap dimensions and still permit direct donor closure.

(ii) Segmental transposition can be done by splitting muscle between its mid and lateral thirds in an effort to retain lateral third of the muscle for function preservation after elevation of the medial two thirds of the muscle with its associated vascular pedicle.

(iii) It is possible to design the rectus muscle with its segmental motor inrervation for functional muscle transposition or transplantation.

(iv) A sensory flap can be designed by dividing the segmental lateral cutaneous sensory nerve fibres during flap elevation.

Precautions have to be observed during elevation of this flap;

(i) A patient who has had prior abdominal incisions where there is a risk of previous division of internal muscle circulation or musculocutaneous perforating vessels between the muscle and abdominal skin. Any portion of a skin island located beyond the rectus muscle with an intervening old incision will generally not have adequate circulation and should not be included in the ski island design.

(ii) In patients with impaired micro circulation like Scleroderma or Raynands disease, the skin island should not be extended excessive distances beyond the primary skin territory of the muscle.

(iii) Prior major vascular surgery and involving the external iliac vessels is a relative contraindication for an inferiorly based rectus muscle flap unless selective arteriography confirms patency of inferior epigastric artery.

(iv) Prior pelvic surgery that may cause adhesions between deep surface of the inferior rectus muscle and pelvic viscera.

(v) A Kocher incision for cholecystectomy an absolute contraindication to the use of ipsi lateral superiorly based rectus made of musculocutaneous flap because the rectus muscle has been divided causing loss of integrity of the internal rectus muscle vascular arcade.

(vi) Closure of anterior rectus sheath resulting in tearing of fascia use of synthetic mesh is recommended. This patient was a candidate for synthetic mesh closure.

(vii) If direct closure of donor site is not possible, a skin graft should be used. 
The rectus abdominis musculocutaneous flap is extremely versatile as long as the dominant pedicle remains intact. Donor site functional deficit is minimal in the immediate post operative period, difficulty in sitting from a recumbent position is experienced. However, function is adequately compensated for by external oblique muscles from six weeks onwards (2).

Elevation of a contralateral rectus abdominis muscle flap in this patient was more difficult because of the fibrosis and distorted anatomy of the rectus sheath arising from previous abdominal surgery. During closure of the donor site, the rectus sheath kept on tearing and ideally a nylon mesh should have been used to prevent an anterior abdominal wall hernia (4).

Primary closure of skin was achieved after extensive undermining of adjacent abdominal skin. This led to partial necrosis of skin at the junction of skin flaps and the wound healed by secondary intention. The loss of common femoral artery after excision of aneurysm excluded use of local thigh muscle flaps and the best option was an extended contralateral rectus abdominis myocutaneous flap.

With the loss of the common femoral artery to the left lower limb, ischaemia and gangrene was expected. This was not the case most likely because of collateral circulation.

\section{ACKNOWLEDGEMENTS}

To the surgical residents rotating in our plastic surgery unit who assisted in the preparation of this patient for theatre and collegues in Cardiothoracic Unit who initially managed the patient before inviting me for wound closure at Kenyatta National Teaching and Referral Hospital.

\section{REFERENCES}

1. Halim A.S. and Zulmi W.B. Extended pedicle rectus abdominis myocutaneous flap for thigh reconstruction. Malaysia J. Plas. Surg. 2004; 11: 86.

2. Grabb's Encyclopedia of flaps. Rectus abdominis musculocutaneous flap. Vol. III pg. 1309.

3. Clinical anatomy. Structure of anterior abdominal wall. 7th Edition. 2003; 55

4. Mathes S.J and Nahai F. Reconstructive surgery principles, anatomy and techniques. 1997; 1043. 